\title{
Addressing the scandal of early death among people with schizophrenia
}

\author{
Philip B. Ward BMedSc PhD
}

— Cite as: CMAJ 2017 September 18;189:E1175-6. doi: 10.1503/cmaj.171010

See related article at www.cmaj.ca/lookup/doi/10.1503/cmaj.161351

I $\mathrm{n}$ a linked research paper, Gatov and colleagues ${ }^{1}$ present clear evidence of a three-fold increase in risk of death for people given a diagnosis of schizophrenia in Ontario. When considered in light of previously reported studies conducted in European and Australian settings, ${ }^{2,3}$ these findings strengthen evidence of a substantial gap in life expectancy between this vulnerable population and general populations in developed countries. A gap in life expectancy of this size for any other group of patients might reasonably be expected to lead to correspondingly substantial public health action to redress the health inequality. However, this does not appear to be the case for people with schizophrenia.

Gatov and colleagues found that cardiovascular disease remains a major driver of increased risk of death among people with schizophrenia. ${ }^{1}$ They list several modifiable risk factors prevalent in this population: high rates of tobacco use, lack of physical activity and high levels of sedentary behaviour, poor diet, and barriers to evidence-based treatment of chronic disease. They also note the weight-enhancing impact of medications that are the cornerstone of treatment for psychotic disorders. Thankfully, accumulating evidence shows that these risk factors can be successfully addressed.

A recent pilot randomized controlled trial (RCT) showed that tailored smoking cessation interventions are feasible and effective in people with schizophrenia. ${ }^{4}$ It is difficult to imagine that real progress can be achieved in addressing the life expectancy gap between people living with schizophrenia and their peers in the general population unless high rates of tobacco use are reduced. However, making a clinical impact will require substantial culture change in mental health services, where smoking has often been viewed as one of the few "pleasures" available to people with schizophrenia. Such attitudes may underpin a reluctance by mental health and primary care clinicians to help patients with schizophrenia achieve tobacco cessation. Yet not addressing tobacco use for this reason could be considered to be "killing with misplaced kindness." However, for those prescribed medication to reduce or cease tobacco use, clear treatment algorithms and guidance are needed on how to adjust psychotropic medication dosages.

\section{KEY POINTS}

- Several studies in developed world settings have shown a large gap in risk of early death between people with schizophrenia and those without mental illness.

- Modifiable cardiac risk factors are prevalent among people with schizophrenia, particularly tobacco use, low physical activity, poor diet and propensity to weight gain on antipsychotic treatment.

- Accumulating evidence shows that smoking cessation, diet and exercise interventions can be used successfully in this population, and programs to prevent weight gain on antipsychotic initiation are promising.

- Policy-makers must urgently address disparities in life expectancy between people with schizophrenia and the general population.

Well-conducted RCTs have also shown that behavioural interventions can be implemented to achieve substantial weight loss in people with schizophrenia who have already experienced substantial weight gain. ${ }^{5,6}$ It is less clear how these interventions can be realistically deployed as part of routine care outside of the trial setting. More optimistically, a recent controlled study among youth with firstepisode psychosis in a community-based psychiatric treatment setting showed that the substantial weight gain typically experienced by those commencing treatment with antipsychotics and mood stabilizers can be prevented by implementing intensive diet and exercise interventions. ${ }^{7}$ The intervention is now being effectively implemented as routine care in a large catchment-based mental health service in southeastern Sydney, Australia. Evaluation of routine implementation is continuing and preliminary data are available. ${ }^{9}$ Preventing weight gain means that future cardiometabolic disease may in turn be prevented, and important secondary benefits in terms of increased fitness ${ }^{8}$ and improved diet quality ${ }^{10}$ may stem from sustained impacts of regular exercise and diet modification.

Improved access to care for chronic disease is another important strategy that could help to address the life expectancy gap in people living with schizophrenia. Many strategies need to be trialled, including embedding specialist medical clinics within mental health settings, and empowering and supporting those living with schizophrenia to become assertive seekers of effective treat- 
ment for chronic illness. A key driver for system-wide change would be the requirement that those charged with the responsibility of overseeing comprehensive health systems regularly report on the physical health outcomes in people living with schizophrenia. Benchmarking against outcomes achieved for those without schizophrenia would provide a strong incentive for holistic efforts to address the scandal of premature death ${ }^{11}$ for people with schizophrenia, which taints our current health service provision systems.

\section{References}

1. Gatov E, Rosella L, Chiu M, et al. Trends in standardized mortality among individuals with schizophrenia, 1993-2012: a population-based, repeated crosssectional study. CMAJ 2017;189:E1177-87.

2. Nielsen RE, Uggerby AS, Jensen SO, et al. Increasing mortality gap for patients diagnosed with schizophrenia over the last three decades-a Danish nationwide study from 1980 to 2010. Schizophr Res 2013;146:22-7.

3. Lawrence D, Hancock KJ, Kisely S. The gap in life expectancy from preventable physical illness in psychiatric patients in Western Australia: retrospective analysis of population based registers. BMJ 2013;346:f2539.

4. Gilbody S, Peckham E, Man MS, et al. Bespoke smoking cessation for people with severe mental ill health (SCIMITAR): a pilot randomised controlled trial. Lancet Psychiatry 2015;2:395-402.

5. Daumit GL, Dickerson FB, Wang NY, et al. A behavioral weight-loss intervention in persons with serious mental illness. N Engl J Med 2013;368:1594-602.
6. Green CA, Yarborough BJ, Leo MC, et al. The STRIDE weight loss and lifestyle intervention for individuals taking antipsychotic medications: a randomized trial. Am J Psychiatry 2015;172:71-81.

7. Curtis J, Watkins A, Rosenbaum S, et al. Evaluating an individualized lifestyle and life skills intervention to prevent antipsychotic-induced weight gain in first-episode psychosis. Early Interv Psychiatry 2016;10:267-76.

8. Vancampfort D, Rosenbaum S, Ward PB, et al. Exercise improves cardiorespiratory fitness in people with schizophrenia: a systematic review and meta-analysis. Schizophr Res 2015;169:453-7.

9. Ward PB, Watkins A, Teasdale S, et al. Replicating positive physical health outcomes for youth with psychosis: Extending the Bondi "keeping the body in mind" program to new FEP settings. Early Interv Psychiatry 2016;10:109.

10. Teasdale SB, Ward PB, Rosenbaum S, et al. Solving a weighty problem: systematic review and meta-analysis of nutrition interventions in severe mental illness. Br J Psychiatry 2017;210:110-8.

11. Thornicroft G. Premature death among people with mental illness. BMJ 2013;346:f2969.

Competing interests: None declared.

Affiliations: School of Psychiatry, University of New South Wales Sydney and Schizophrenia Research Unit, Ingham Institute for Applied Medical Research, Liverpool, New South Wales, Australia

This article was solicited and has not been peer reviewed.

Correspondence to: Philip Ward, p.ward@unsw.edu.au 